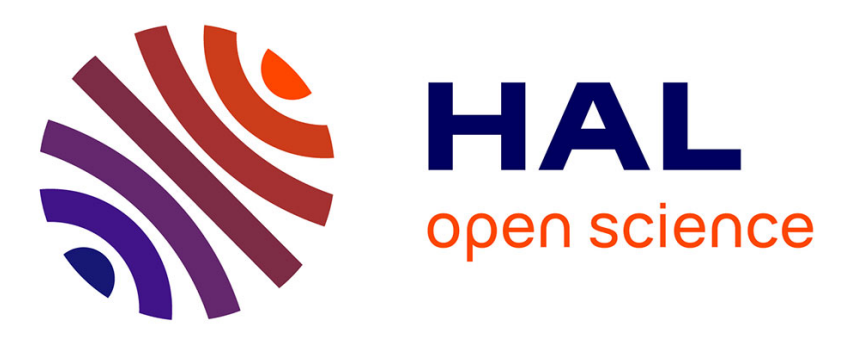

\title{
Operator splitting-based reactive transport models in strong feedback of porosity change: the contribution of analytical solutions for accuracy validation and estimator improvement
}

Vincent Lagneau, Jan van Der Lee

\section{To cite this version:}

Vincent Lagneau, Jan van Der Lee. Operator splitting-based reactive transport models in strong feedback of porosity change: the contribution of analytical solutions for accuracy validation and estimator improvement. Journal of Contaminant Hydrology, 2009, 112 (1-4), pp.118-129. 10.1016/j.jconhyd.2009.11.005 . hal-00505026

HAL Id: hal-00505026

https://hal-mines-paristech.archives-ouvertes.fr/hal-00505026

Submitted on 22 Jul 2010

HAL is a multi-disciplinary open access archive for the deposit and dissemination of scientific research documents, whether they are published or not. The documents may come from teaching and research institutions in France or abroad, or from public or private research centers.
L'archive ouverte pluridisciplinaire HAL, est destinée au dépôt et à la diffusion de documents scientifiques de niveau recherche, publiés ou non, émanant des établissements d'enseignement et de recherche français ou étrangers, des laboratoires publics ou privés. 


\title{
Operator splitting-based reactive transport models in strong feedback of porosity change: the contribution of analytical solutions for accuracy validation and estimator improvement.
}

\author{
Vincent Lagneau $^{\mathrm{a}, *}$ Jan van der Lee ${ }^{\mathrm{b}}$ \\ a Mines ParisTech, 35 rue Saint Honoré, 77305 Fontainebleau Cedex, France \\ ${ }^{\mathrm{b}}$ The Materials Ageing Institute, EDF R\&D, Site des Renardières, 77818 \\ Moret-sur-Loing Cedex, France
}

\begin{abstract}
Reactive transport is a highly non-linear problem requiring the most efficient algorithms to rapidly reach an accurate solution. The non-linearities are increased and the resolution is even more demanding and CPU-intensive when considering feedback of dissolution or precipitation reactions on hydrodynamic flow and transport, commonly referred to as the variable porosity case. This is particularly true near clogging, which leads to a very stiff systems and therefore small time-steps. The operator-splitting approach is often cited is a widely use method to solve these problems: it consists in solving sequentially the transport then the chemistry part of the problem. Operator-splitting appears to be an accurate approach, provided that the solution is iteratively improved at each time-step.

The paper details analytical solutions and test-cases for this class of problems. They demonstrate that iterative improvement is then compulsory. They also helped develop an improved estimator/corrector method which allows to reach convergence faster and to reduce stiffness. The efficiency improvement is significant as illustrated by an example of carbonation of a cement paste, a well-known problem that leads to complete clogging of the interface layer.
\end{abstract}

Key words: reactive transport, chemistry, feedback, variable porosity, clogging, analytical solutions.

* Corresponding author: vincent.lagneau@mines-paristech.fr 


\section{Introduction}

Simulation of hydrodynamic transport of strongly reactive chemicals is generally computationally intensive and sometimes even prohibitively CPU-consuming (21). The usual modeling approach consists in solving the problem at the scale of the Representative Elementary Volume (REV, (4)), i.e. on a scale large enough to average out details of the pore structure. One of the benefits of this approach is that it can tackle relatively large systems within reasonable CPU limits. Examples include simulations of radioactive waste repositories (13), stabilization of industrial wastes (12) and aquifer contamination $(8 ; 23)$, to cite only a few.

Dissolution and precipitation of solid phases tend to modify the volume and the structure of the porous medium, thus modifying flow and transport properties (17). Modification of these properties cannot be explicitly simulated by models based on the REV scale. They can be included, however, by means of geometrical assumptions (simplified geometries for the porous medium, $(19 ; 16 ; 17)$ ) or empirical laws (e.g. Kozeny-Carman for permeability $(5 ; 9)$, or Archie's law of diffusion $(2 ; 9))$. No general relationship exists, and the field is still an active research area.

The feedback of chemical reactions on transport properties adds another nonlinearity to the system, requiring efficient algorithms to reach an accurate solution in reasonable computer time. The correct simulation of such feedback systems is fundamental in numerous domains. For instance, some radioactive waste geological storage scenarios involve clay-cement interfaces, which are known to lead to a strong local reduction of the porosity $(25 ; 11)$; failing to take this phenomenon into account can seriously damage the quality of predictive simulations. Other domains include for instance slag-heap leaching (where pyrite oxidation can lead to the formation of high molar volume gypsum, e.g. (15)) or transport in fractures (whose opening or closure are controlled by calcite plugging, e.g. (20)).

The literature displays a variety of applications performed with several reactive transport codes, including chemical feedback on transport, over the last 15 years (e.g. $(24 ; 28 ; 14 ; 22))$. However, efficiency and accuracy are still two keys for these CPU-demanding resolutions. In this paper, the authors want to precisely detail the coupling algorithms, with respect to both aspects, with a focus on simulation with significant feedback of chemistry on hydrodynamics. The first aim is to ensure accuracy, which is of the utmost importance if reactive transport simulations are to be trusted for operational use. Then, the efficiency issue is addressed with a view to keep resolution times at a reasonable level. Throughout the process, applications are performed with the versatile reactive transport code HYTEC (27), developed at Mines-ParisTech, 
and based on a sequential iterative algorithm whose formalism and equations are displayed in section 2. Note however that the validation and the improvements proposed on the sequential iterative approach would hold for any code based on this formalism.

A methodology is developed to test the validity of the algorithms: two simple problems, with analytical solutions, are examined; comparing simulation results to the analytical solutions offers a means of choosing the most suited method. After the validation of the algorithm, some general observations show a way to fine-tune it to improve its speed of convergence (section 3). Finally, in section 4, an application with a strong feedback of porosity is proposed: the simulation of cement carbonation. Beyond the simulation results, the effect of the optimization is monitored as compared to the natural algorithm.

\section{Verification of the accuracy of the algorithm}

\subsection{Theory of the sequential approach with feedback of chemistry on transport}

Two main classes of algorithms are found in the literature: global implicit and operator-splitting $(29 ; 6)$. The latter, which consists in the sequential resolution of the transport- and the chemical-part of the systems, has several advantages: among which are the reduction of the size of the systems and the possibility to develop the resolution modules separately (27). Here we focus on the operator-splitting with iterative improvement: transport and chemistry are repeatedly solved one after the other until convergence is reached with respect to some fixed point. The method guarantees the accuracy of the solution without time-step criteria (29).

The necessity to solve the coupled flow problem following an implicit approach is not discussed in this paper. It seems unnecessary due to the difference in relaxation time for the flow equation on the one hand, and the chemical and transport equations on the other. It is therefore sufficient to solve the flow equation at the beginning of the time-step, then to iteratively solve the chemistry, transport and porosity problem. At the end of the time-step, the permeability field is updated according to porosity changes, to prepare for the resolution of the flow problem at the next time-step.

The usual approach to solving chemical systems is based on the principal components formalism (18): the unknowns are limited to the total concentrations $\tilde{c}_{j}$ of a limited number of basis species (components). The concentration of all the species in the system can be computed with the chemical operator

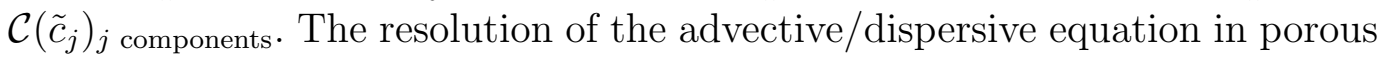


media is then required for all the components $(10 ; 18)$ :

$$
\begin{aligned}
\frac{\partial \omega c}{\partial t} & =\mathcal{T}(c)-\frac{\partial \omega \bar{c}}{\partial t} \\
\mathcal{T}(c) & =\operatorname{div}\left(D_{e} \cdot \overrightarrow{\operatorname{grad}} c-c \cdot \vec{U}\right)
\end{aligned}
$$

where $\omega$ is the porosity, $D_{e}$ the effective diffusion/dispersion coefficient (as opposed to $D=D_{e} / \omega$ the pore diffusion), and $\vec{U}$ the local filter velocity. The transport operator can be noted $\mathcal{T}$. In this equation, we separate the mobile part of the total concentration $c$ (aqueous complexes, colloids) from the immobile part $\bar{c}$ (minerals, surface complexation, etc). Finally, the evolution of the porosity is obtained by a balance of the volumes of all the minerals in the system:

or equivalently

$$
\omega=1-\sum_{m \text { minerals }} V_{m} \omega c_{m}
$$

$$
\frac{\mathrm{d} \omega}{\omega}=-\frac{\mathrm{d} f}{1+f} \quad \text { with } f=\sum_{m \text { minerals }} V_{m} c_{m}
$$

where $V_{m}$ is the molar volume $\left(\mathrm{L} \cdot \mathrm{mol}^{-1}\right)$ and $c_{m}$ the concentration of mineral $m$ in $\mathrm{mol} \cdot \mathrm{L}^{-1}$ of solution. The concentration in $\mathrm{mol} \cdot \mathrm{L}^{-1}$ of medium, $C_{m}$, is obtained as follows:

$$
C_{m}=\omega c_{m}
$$

The quantity $C_{m}$ does not depend on the porosity, and can only change if the mineral $m$ dissolves or precipitates. On the contrary, $c_{m}$ evolves with mineral reactions, but also with porosity changes (even if $m$ is a non-reactive mineral).

The sequential resolution of these equations consists in numerically solving the transport, then the chemistry. Accuracy is insured by iterating until the model converges to a fixed point. We thus build a sequence for all the components $\left(\tilde{c}_{j}^{t+\delta t, p}, c_{j}^{t+\delta t, p}, \bar{c}_{j}^{t+\delta t, p}\right)_{j \text { components, }}$, where $p=2 i+1$ after a transport step and $p=2 i+2$ after a chemistry step:

(1) resolution of the transport for one time-step:

$$
\frac{\omega^{\zeta} c_{j}^{t+\delta t, 2 i+1}-\omega^{t} c_{j}^{t}}{\delta t}=\mathcal{T}\left(c_{j}\right)-\frac{\omega^{\zeta} \bar{c}_{j}^{t+\delta t, 2 i}-\omega^{t} \bar{c}_{j}^{t}}{\delta t}
$$

where $\left(c_{j}^{t}, \bar{c}_{j}^{t}\right)$ is the solution at the current time, $\left(c_{j}^{t+\delta t, 2 i}, \bar{c}_{j}^{t+\delta t, 2 i}\right)$ is the solution of the chemical module for the new time at the previous iteration, and $\zeta$ stands for the time discretization for the porosity (see below).

(2) resolution of the speciation, using the local values of the total concentrations as given by the transport operator: $\mathcal{C}\left(c_{j}^{t+\delta t, 2 i+1}+\bar{c}_{j}^{t+\delta t, 2 i}\right)_{\forall j}$. The speciation allows us to discriminate between fixed and mobile fractions 
(for transport purposes), and to calculate the mineral volumes (to feed the porosity equations 1-2).

Equation 3 shows that the concentration of the minerals (even inert ones) evolves due to porosity changes. For consistency's sake, the mineral concentrations must therefore be updated to follow porosity evolutions. Porosity appears in equation 4: directly in the accumulation term, and indirectly through the diffusion/dispersion coefficient and the velocity field. It is important to determine whether the porosity can be used explicitly (i.e. updated after each time step only, $\zeta=(t)$ ) which is less CPU consuming: the transport parameters are then updated less frequently, and the transport matrices have only to be recalculated at each time step rather than each coupling iteration. Or alternatively, if a more robust implicit approach is required: $\zeta=(t+\delta t, 2 i)$. To answer this question, we developed two simple problems and their subsequent analytical solutions. Confronting the explicit/implicit choices in these analytical solutions will be conclusive as to the accurate algorithm.

\subsection{Reaction-only test case}

The first case developed for this study can be solved by using the reactivetransport formalism although no actual transport is involved. Diffusion is eliminated by insuring the absence of concentration gradients, i.e. appropriate initial and boundary conditions. The system contains a single mineral A(m) (of concentration $\bar{c}$ ), consisting of a single component $\mathrm{A}(\mathrm{aq})$ (of concentration $c$ ). The growth of mineral $\mathrm{A}(\mathrm{m})$ is simulated with the following kinetic law:

$$
\left\{\begin{aligned}
\mathrm{A}(\mathrm{m}) & \rightleftharpoons \mathrm{A}(\mathrm{aq}) \\
\frac{\mathrm{d} \omega \bar{c}}{\mathrm{~d} t} & =-k S_{s}(\omega)\left(1-\frac{c}{K}\right) \\
\frac{\mathrm{d} \omega c}{\mathrm{~d} t} & =-\frac{\mathrm{d} \omega \bar{c}}{\mathrm{~d} t}
\end{aligned}\right.
$$

where $K$ is the solubility constant of the mineral, $k$ the kinetic rate $\left(\mathrm{mol} \cdot \mathrm{m}^{-2} \cdot \mathrm{s}^{-1}\right)$, and $S_{s}$ the specific surface area $\left(\mathrm{m}^{2} \cdot \mathrm{m}^{-3}\right.$ of rock).

The overall system (for a water-saturated media) is described by the following system of equations:

$$
\left\{\begin{aligned}
\frac{\mathrm{d} \omega c}{\mathrm{~d} t} & =-\frac{\mathrm{d} \omega \bar{c}}{\mathrm{~d} t} & & \text { (conservation of mass) } \\
& =\frac{1}{V_{m}} \frac{\mathrm{d} \omega}{\mathrm{d} t} & & \text { (conservation of volume) }
\end{aligned}\right.
$$

The detailed resolution, given in appendix A, is based on the direct substitution so that the porosity remains the only variable of the system, and uses a 


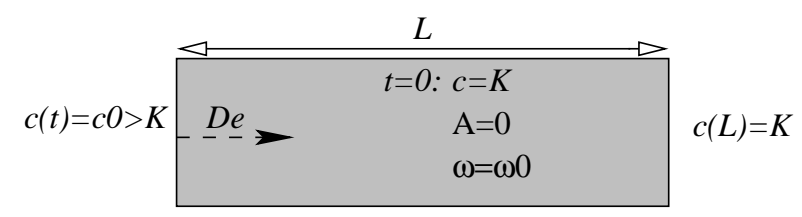

Fig. 1. Schematic representation of the second analytical problem.

simple relationship between porosity and the evolution of the specific surface area. The overall solution is then:

$$
c(t)=\frac{1 / V_{m}-K \zeta(t)}{1-\zeta(t)}
$$

where $\zeta(t)=\frac{c_{0}-1 / V_{m}}{c_{0}-K} \exp \left[-\frac{k}{K}\left(K V_{m}-1\right) S_{0} t\right]$

$$
S_{s}(\omega)=\omega S_{0}
$$

\subsection{Reactive-transport test case}

A second simple problem is proposed to test the behavior of the proposed algorithm in the presence of (diffusive) transport. The chemical system is the same as before, and a diffusive transport component has been added. The system is described by the following equations:

$$
\left\{\begin{array}{rlrl}
\mathrm{A}(\mathrm{m}) & \rightleftharpoons \mathrm{A}(\mathrm{aq}) & & \text { with } K=c_{\text {equil. }} \\
\frac{\mathrm{d} \omega c}{\mathrm{~d} t} & =k S_{s}(1-c / K) & & \\
\frac{\partial \omega c}{\partial t} & =\frac{\partial}{\partial x}\left(D_{e}(\omega) \frac{\partial c}{\partial x}\right)-\frac{\partial \omega \bar{c}}{\partial t} &
\end{array}\right.
$$

We carefully chose the initial and boundary conditions to simplify the resolution (Fig. 1): initial solute concentration at equilibrium with $\mathrm{A}(\mathrm{m})$, oversaturation imposed at the inlet. The system is therefore always at equilibrium or over-saturated, which eliminates non-linearities due to the presence or absence of a mineral in an under-saturated state.

The system follows the system of equations:

$$
\left\{\begin{array}{l}
\frac{\partial \omega c}{\partial t}=\frac{\partial}{\partial x}\left(D_{e}(\omega) \frac{\partial c}{\partial x}\right)-\frac{\partial \omega \bar{c}}{\partial t} \\
\frac{\partial \omega \bar{c}}{\partial t}=-k S(\omega)\left(1-\frac{c}{K}\right)=-\frac{1}{V_{m}} \frac{\partial \omega}{\partial t}
\end{array}\right.
$$

As in the previous problem, we need to inject information relative to the evolution of the pore structure. To keep the system simple, we used $S(\omega)=$ $\omega S_{0}$ and $D_{e}(\omega)=\omega D_{0}$. 
The resolution is detailed in appendix B: it relies on a simplification of the system, valid under small local gradients of porosity, which reduces the system to a ODE equation of the aqueous concentration only. Porosity and mineral concentrations are recovered in an independent step. Although quite complex (Eq. B.4), the solution can be further simplified under quasi stationary state (transport fast compared to chemistry), and yields the asymptotically simplified solution:

$$
\left\{\begin{array}{l}
c(x, t)=A e^{\Omega x}+B e^{-\Omega x}+K \\
\omega(x, t)=\omega_{0} \exp \left[-V_{m} k S_{0} / K\left(A e^{\Omega x}+B e^{-\Omega x}\right) t\right]
\end{array}\right.
$$

with $A=\frac{c_{0}-K}{1-e^{2 \Omega L}}, B=\frac{c_{0}-K}{1-e^{-2 \Omega L}}$ and $\Omega=\sqrt{k S_{s_{0}} /\left(K D_{0}\right)}$.

\subsection{Implicit or explicit approach: model validation}

The sequential iterative algorithm was implemented in the reactive code HYTEC, using the two methods: explicit $(\zeta=(t))$ or implicit $(\zeta=(t+\delta t, 2 i))$ reference to the porosity in the discrete transport equation 4. Moreover, a third, crude method was tested, where the mineral concentrations (in $\mathrm{mol} \cdot \mathrm{L}^{-1}$ of solution) were not updated for porosity changes.

The numerical results were compared to the analytical solutions in the two problems. Realistic parameters were chosen, by assuming a medium composed of quartz in a simplified silica system. A high initial concentration in $A(a q)$ was chosen so that the system remained oversaturated:

$$
\begin{cases}K & =0.1\left(\text { as per } \mathrm{mol} \cdot \mathrm{m}^{-3}, \text { i.e. } c=10^{-4} \mathrm{~mol} \cdot \mathrm{L}^{-1} \text { at equilibrium }\right) \\ k & =2 \times 10^{-8} \mathrm{~mol} \cdot \mathrm{m}^{-2} \cdot \mathrm{s}^{-1} \\ V_{m} & =4 \times 10^{-4} \mathrm{~m}^{3} \cdot \mathrm{mol}^{-1} \\ S_{0} & =10^{4} \mathrm{~m}^{2} \cdot \mathrm{m}^{-3} \text { of porous medium } \\ c(0) & =1000 \mathrm{~mol} \cdot \mathrm{m}^{-3}\end{cases}
$$

The transport parameters were arbitrarily to $D_{0}=10^{-10} \mathrm{~m}^{2} \cdot \mathrm{s}^{-1}$ and $\omega(0)=$ 0.79 .

Figure 2 shows the analytical results compared with the different numerical methods for the reaction-only test case. It is immediately clear that the crude method leads to wrong results (even for inert minerals). The explicit method is also in error with an amplitude about half of that of the crude method. Only the implicit method gives excellent results, so that we now focus on this 

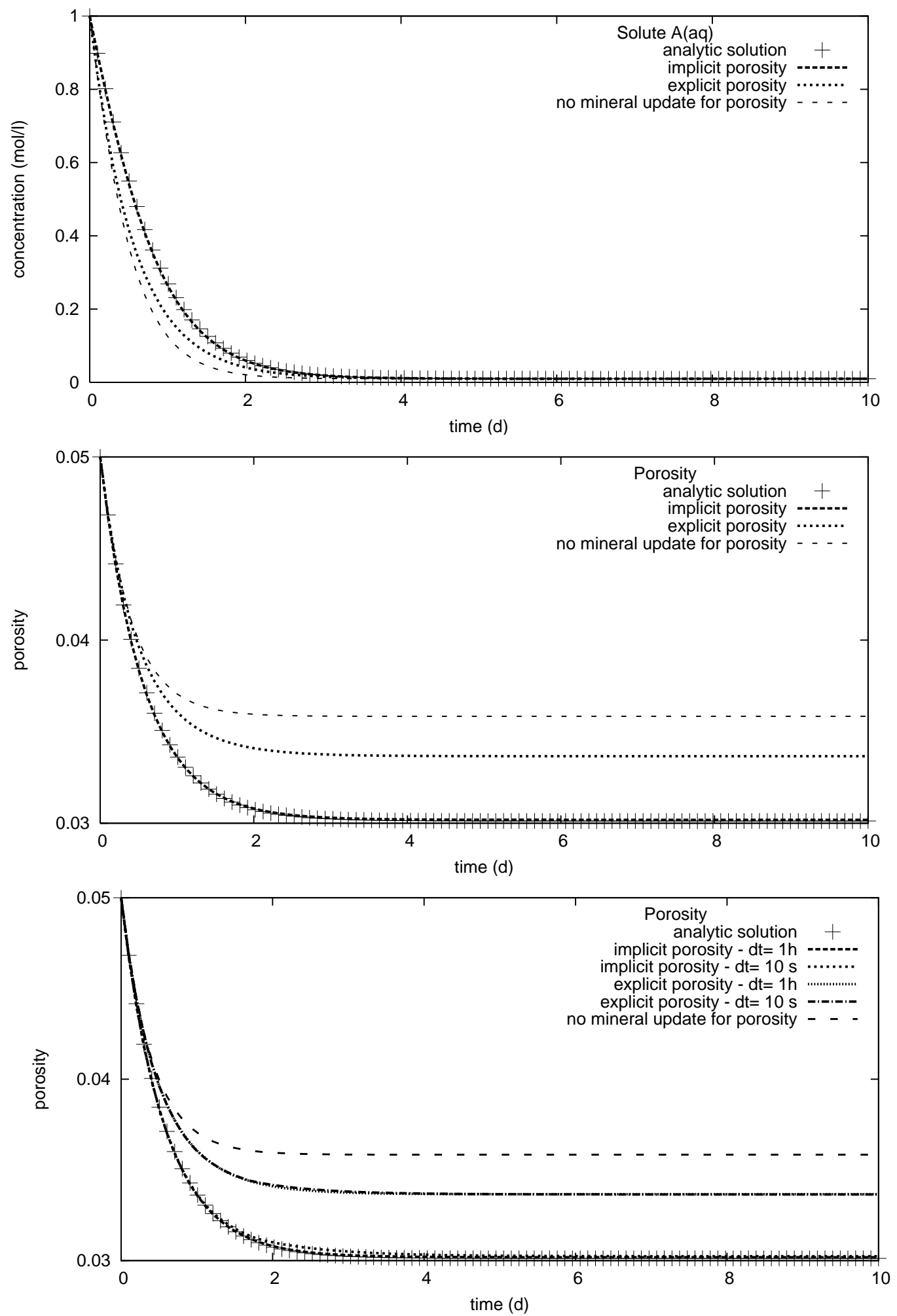

Fig. 2. Simple system without transport. Illustration of the evolution of solute concentration (top) and porosity (middle) for the analytical solution and three resolution algorithms: the crude method without mineral concentration update for porosity, explicit and implicit porosity updates. The effect of the time step is given with simulations using constant time step $1 \mathrm{~h}$ and $10 \mathrm{~s}$ for both formulation (bottom). 


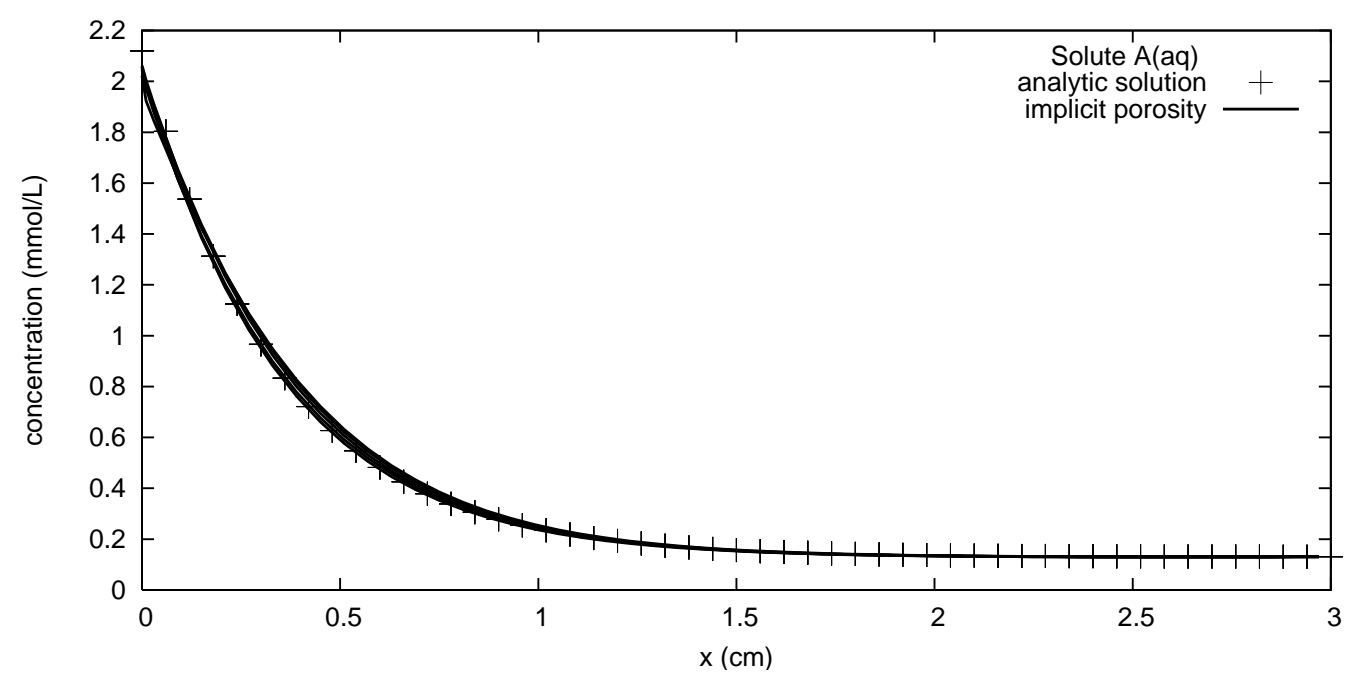

Fig. 3. Second analytical problem, reactive transport, solute profile; the quasi stationary state analytical solution, and HYTEC numerical solution algorithm (using the implicit algorithm for porosity feedback) for five different values of time: the solute profile is indeed quasi independent of time.

method only. Curiously, reducing the time step does not seem to improve the accuracy of the explicit (non-iterative) formulation (Fig. 2, bottom).

However, we still have to demonstrate that the implicit approach is correct in really coupled transport and chemistry problems. Figure 3 shows the comparison between the analytical and the implicit resolution results for the reactive transport test case. The solute concentration profiles are superimposed for five different values of time, demonstrating that the quasi stationary state approximation for the solute concentrations is valid. The predicted profiles of mineral $\mathrm{A}(\mathrm{m})$ and the porosity are in very good agreement with the analytical solution as illustrated by Fig. 4. As anticipated, the agreement with the analytical solution deteriorates slightly for higher values of time: indeed, as the spatial gradient of porosity builds up, the approximation on the cross-term of Eq. B.2 becomes invalid, introducing errors in the analytical solution.

The conclusions from the two analytical problems are straightforward: an accurate solution requires a method which takes the porosity change into account implicitly, i.e. at each coupling iteration. Accordingly, the correct coupling algorithm completes the scheme outlined 2.1:

(1) Transport resolution:

$$
\forall \text { components } j, \frac{\omega^{t+\delta t, 2 i} c_{j}^{t+\delta t, 2 i+1}-\omega^{t} c_{j}^{t}}{\delta t}=\mathcal{T}\left(c_{j}\right)-\frac{\omega^{t+\delta t, 2 i} \bar{c}_{j}^{t+\delta t, 2 i}-\omega^{t} \bar{c}_{j}^{t}}{\delta t}
$$



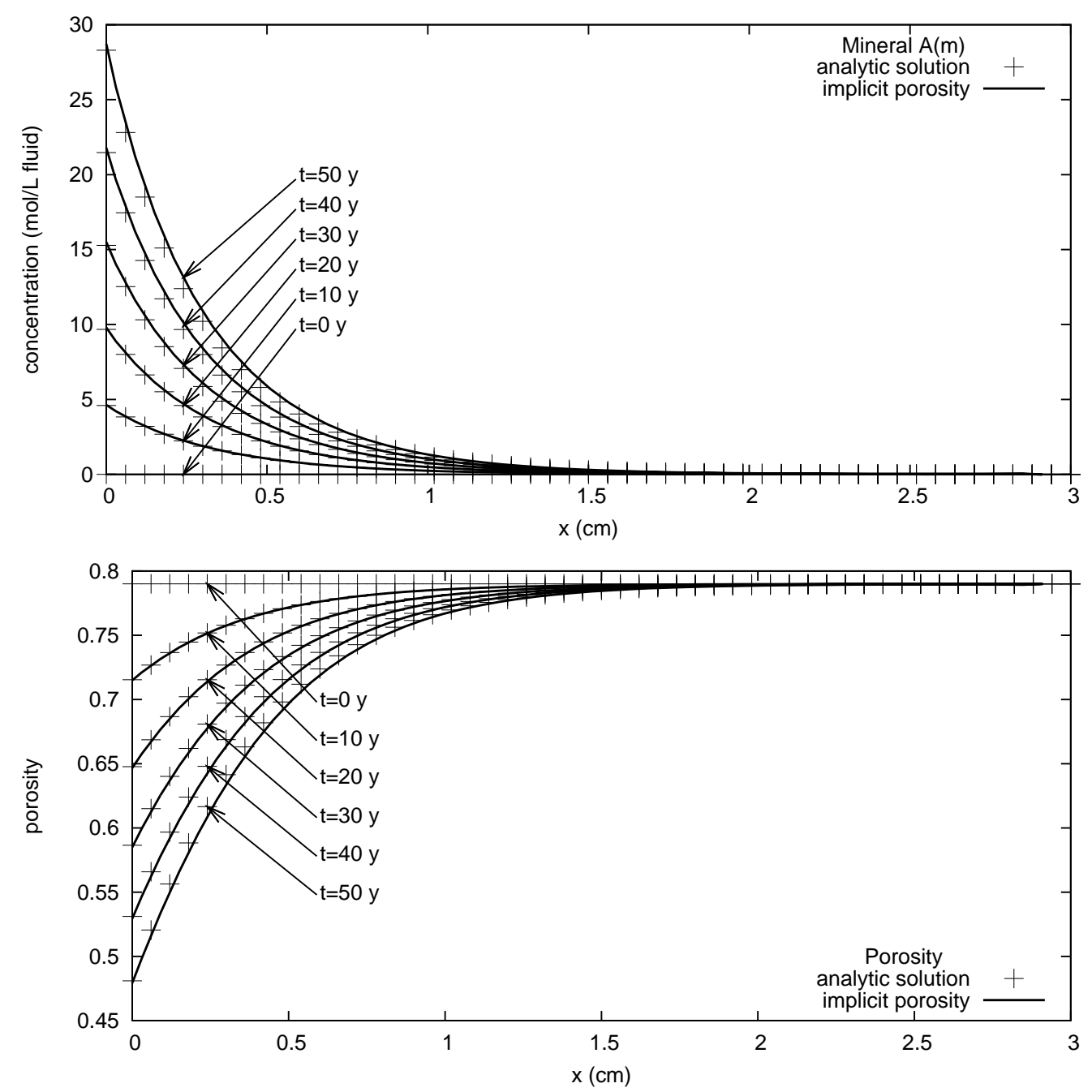

Fig. 4. Second analytical problem, reactive transport, mineral (top) and porosity (bottom) profiles for five different values of time: analytical solution and numerical solution by HYTEC, using the implicit algorithm for porosity feedback.

(2) Chemical speciation:

$$
\forall \text { nodes, } \mathcal{C}\left(c_{j}^{t+\delta t, 2 i+1}+\bar{c}_{j}^{t+\delta t, 2 i}\right)_{\forall j}
$$

provides the speciation and in particular the mineral concentrations, the total fixed concentrations $\bar{c}_{i}^{t+\delta t, 2 i+2}$

(3) Modification of the porosity:

$$
\forall \text { nodes, } \omega^{t+\delta t, 2 i+2}=f\left(c_{m}^{t+\delta t, 2 i+2}\right)
$$

and update of the mineral concentrations (in $\mathrm{mol} \cdot \mathrm{L}^{-1}$ of solution) to accommodate the porosity change. 
Steps 1-3 must be repeated until the fixed point is reached:

$$
\text { test } \forall j,\left\|\bar{c}_{j}^{t+\delta t, 2 i+2}-\bar{c}_{j}^{t+\delta t, 2 i}\right\|_{\infty}<\varepsilon \times\left\|\bar{c}_{j}^{t+\delta t, 2 i}\right\|_{\infty}
$$

where $\varepsilon$ is the precision of coupling, and $\|\cdot\|_{\infty}$ refers to the spatial discretization. The convergence test is performed after step 3, using the fixed fractions $\bar{c}_{j}$. Convergence is immediate for mobile species, whose precise speciation does not modify the total mobile fraction for the transport step, thereby not interfering with the convergence of the coupling.

This algorithm is the base of the HYTEC resolution scheme. It also includes a heuristic control of the time-step, based on the number of iterations required to reach convergence. If this number is larger than a user-defined threshold (default value 20), HYTEC continues with the resolution with a time-step decreased by an user-defined increment (default 5\%); alternatively, if convergence is reached faster, the time-step is increased by the same increment. When convergence is not reached after a limit (default 60 iterations), HYTEC considers a failed convergence, goes back one time-step before resuming the resolution with a $30 \%$ decreased time-step. All through the simulation, the time-step remains bounded by the Courant criterion, which can be made sloppier by the user for semi-implicit or implicit time-discretization schemes for the transport.

\section{Optimization of the algorithm}

The previous sections have demonstrated that an iteratively improved sequential approach is accurate for problems with feedback of chemistry on transport, provided porosity changes are taken into account implicitly. The latter condition has a CPU consumption penalty. In this section, it is shown how CPU time can be reduced by a careful choice of the starting point (or estimator) of the fixed-point algorithm.

Remember that the algorithm consists in finding, for each time-step, the fixed point of the sequence $\left(\left(c_{j}^{t+\delta t, p}\right)_{j \text { component }},\left(\bar{c}_{j}^{t+\delta t, p}\right)_{j \text { component }}, \omega^{t+\delta t, p}\right)$ using equations 9-11. Just like any fixed-point algorithm, the starting point plays a role in the speed of convergence: any value (close enough to the solution) is acceptable, provided that porosity and concentrations are consistent. A natural choice, though not necessarily the best, is to use the result of the previous time-step as the starting point for the calculations:

$$
\left\{\begin{aligned}
\bar{c}^{t+\delta t, 0} & =\bar{c}^{t} \\
\omega^{t+\delta t, 0} & =\omega^{t}
\end{aligned}\right.
$$




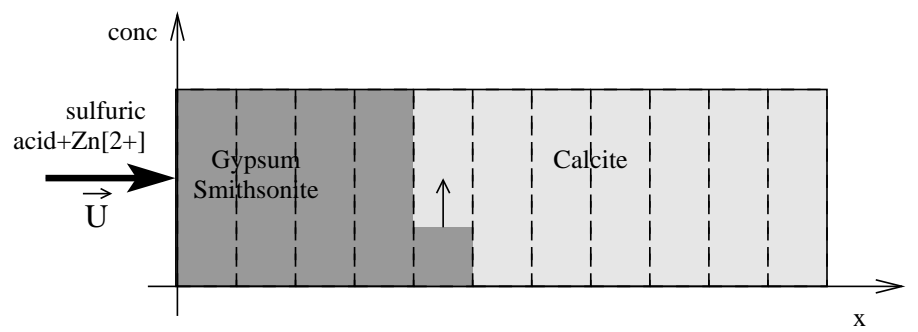

Fig. 5. Schematic of the simple model to test the optimization of the fixed-point initial estimator.

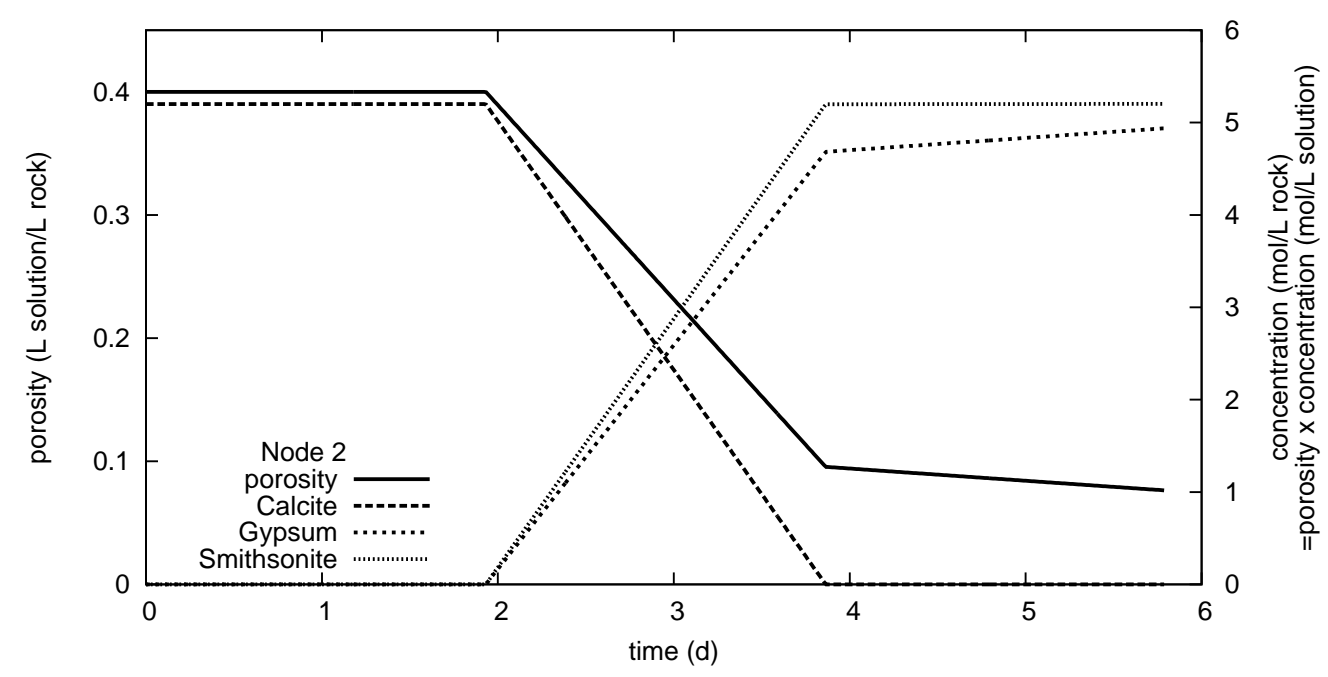

Fig. 6. HYTEC results of the simple model to test the optimization of the fixed-point initial estimator: evolution of the minerals and porosity in a single cell.

To investigate this point, we define a simple test system. We consider a column of porous medium containing calcite and flushed with a sulphuric-acid solution containing zinc (fig. 5). The considered transport mechanisms are advection, dispersion and diffusion. Furthermore, we assume thermodynamic equilibrium conditions; hence, calcite is dissolved and, due to the input of sulphates and zinc, gypsum and smithsonite are formed. These minerals increase significantly the total mineral volume of the column (17):

$$
\text { Calcite }+\mathrm{Zn}^{2+}+\mathrm{SO}_{4}{ }^{2-} \rightleftharpoons \text { Gypsum }+ \text { Smithsonite. }
$$

The simulations show the formation of a reacting front, associated with a decrease in porosity. A closer look at the numerical behavior of the system (Fig. 6), reveals that the front changes cells only (at first glance) when the calcite is completely exhausted.

The evolution of porosity on the one hand, and of the product porosity by mineral concentrations (in $\mathrm{mol} \cdot \mathrm{L}^{-1}$ of solution) on the other hand, are linear in time. This observation can be reproduced easily with an analytical resolution of the discrete system. Based on this observation, an improved estimator can 
be proposed, i.e.:

$$
\left\{\begin{array}{c}
\frac{\Delta_{t \rightarrow(t+\delta t, 0)}(\omega)}{\delta t}=\frac{\Delta_{\left(t-\delta t^{\prime}\right) \rightarrow t}(\omega)}{\delta t^{\prime}} \\
\frac{\Delta_{t \rightarrow(t+\delta t, 0)}(\omega \bar{c})}{\delta t}=\frac{\Delta_{\left(t-\delta t^{\prime}\right) \rightarrow t}(\omega \bar{c})}{\delta t^{\prime}}
\end{array}\right.
$$

or, more precisely:

$$
\left\{\begin{array}{l}
\omega^{t_{\kappa+1}, 0}=\omega^{t_{\kappa}}+\frac{t_{\kappa+1}-t_{\kappa}}{t_{\kappa}-t_{\kappa-1}}\left(\omega^{t_{\kappa}}-\omega^{t_{\kappa-1}}\right) \\
\bar{c}^{t_{\kappa+1}, 0}=\frac{\omega^{t_{\kappa}}}{\omega^{t_{\kappa+1}, 0}} \bar{c}^{t_{\kappa}}+\frac{t_{\kappa+1}-t_{\kappa}}{t_{\kappa}-t_{\kappa-1}} \frac{\omega^{t_{\kappa}} \bar{c}^{t_{\kappa}}-\omega^{t_{\kappa-1}} \bar{c}^{t_{\kappa-1}}}{\omega^{t_{\kappa+1}, 0}}
\end{array}\right.
$$

This starting point provides a (much) closer estimate of the solution. In the above problem, this estimator is actually exact as long as the front does not change cells.

\section{Application: cement carbonation}

After the demonstration on this specific test-case, we applied the iterative algorithm, with the improved estimator, on more complex systems. The difference in computational effort for the normal and the improved estimators will therefore be highlighted. One such system is the simulation of cement carbonation: the problem is inherently difficult for reactive transport codes due to strongly coupled chemical and hydrodynamic processes. The hydrolysis of the cement phases (hydrated calcium silicates CSH, calcium sulpho-aluminates) leads to a release of calcium which diffuses towards the interface; there, it reacts with the carbonates of the leaching solution. Accordingly, a thin but nearly non-porous calcite crust forms at the interface $(3 ; 26)$.

HYTEC, with the implicit algorithm and the improved estimator, was used to simulate the case of a simplified Portland type cement (CEM I), where the CSH gel continuum is represented by three discrete characteristic minerals with decreasing $\mathrm{Ca} / \mathrm{Si}$ ratio: $1.8,1.1$, and 0.8 (Tab. 1). This simplified description of the gel continuum has been introduced by (1): each discrete $\mathrm{CSH}$ mineral equivalent accounts for a behavioral zone of the CSH gel in the $\mathrm{Ca}-\mathrm{Si}-\mathrm{pH}$ graph; the higher the calcium content, the higher the equilibrium $\mathrm{pH}$. Initial cements, containing portlandite, have a high $\mathrm{pH}$ (around 12.5), and their hydrolysis leads to lower calcium-content phases and lower equilibrium $\mathrm{pH}$.

In this model, degradation is due to the contact with a fluid at $\mathrm{pH}=7$ and $\left[\mathrm{HCO}_{3}^{-}\right]=10^{-3}$ molal. As expected under these conditions the cement 


\begin{tabular}{lllll}
\hline mineral & formula & $\log K$ & molar volume \\
\hline Portlandite & $\rightleftharpoons \mathrm{Ca}^{2+}-2 \mathrm{H}^{+}+2 \mathrm{H}_{2} \mathrm{O}$ & -22.56 & $33.1 \mathrm{ml} \cdot \mathrm{mol}^{-1}$ \\
CSH1.8 & $\rightleftharpoons 1.8 \mathrm{Ca}^{2+}-3.6 \mathrm{H}^{+}+\mathrm{SiO}_{2}(\mathrm{aq})+3.6 \mathrm{H}_{2} \mathrm{O}$ & -32.58 & $86.7 \mathrm{ml} \cdot \mathrm{mol}^{-1}$ \\
$\mathrm{CSH} 1.1$ & $\rightleftharpoons 1.1 \mathrm{Ca}^{2+}-2.2 \mathrm{H}^{+}+\mathrm{SiO}_{2}(\mathrm{aq})+2.2 \mathrm{H}_{2} \mathrm{O}$ & -17.00 & $63.5 \mathrm{ml} \cdot \mathrm{mol}^{-1}$ \\
$\mathrm{CSH} 0.8$ & $\rightleftharpoons 0.8 \mathrm{Ca}^{2+}-1.6 \mathrm{H}^{+}+\mathrm{SiO}_{2}(\mathrm{aq})+1.6 \mathrm{H}_{2} \mathrm{O}$ & -11.07 & $53.5 \mathrm{ml} \cdot \mathrm{mol}^{-1}$ \\
Calcite & $\rightleftharpoons \mathrm{Ca}^{2+}-2 \mathrm{H}^{+}+\mathrm{HCO}_{3}^{-}$ & -1.85 & $36.9 \mathrm{ml} \cdot \mathrm{mol}^{-1}$ \\
\hline
\end{tabular}

Table 1

Chemical properties of the mineral phases in the cement carbonation simulation.

hydrolyzes with a characteristic sequence of mineral fronts with a decreasing calcium content and $\mathrm{pH}$ (Fig.7). The overall result is an increase in the porosity in the profile for more degraded cement. The calcium leached from the degraded cement diffuses towards the interface with the attack solution, where the carbonate content allows for the precipitation of a thin calcite crust. Calcium is thus re-concentrated locally from the larger leached area, leading to a local and sharp decrease of the porosity. The system is numerically hard to handle, since it leads to a very high concentration of calcite (up to $27 \mathrm{~mol} \cdot \mathrm{L}^{-1}$ of rock) and a porosity which drops down to nearly 0 . As a result, the mineral concentration expressed in $\mathrm{mol} \cdot \mathrm{L}^{-1}$ of solution (concentration in $\mathrm{mol} \cdot \mathrm{L}^{-1}$ of rock divided by the porosity) tends towards infinity and introduces a numerical instability of type $0 \times \infty$ in the accumulation term $\delta \omega \bar{c} / \delta t$.

The performance of the simple estimator (Eq. 12) and of the improved one (Eq. 13) was monitored via the number of coupling iterations required to reach convergence at each time-step. The improved estimator requires a single iteration per time-step, except at some points when the front moves to an adjacent cell: here, the number of iterations reaches about 20. Indeed, when the front changes cells, the mineral assemblage changes in two cells over the time-step: complete depletion of the primary mineral in the first cell, creation of new secondary minerals in the second cell. In these conditions, the improved estimator leads to inaccurate predictions, and iterations are required to reach convergence.

On the contrary, the simple estimator constantly requires about 20 iterations, except at times when the fronts change cells, in which case it is reduced to a few iterations (note that the number of 20 iterations is a consequence of the code time-step management: HYTEC automatically reduces the time-step as soon as the number of iterations exceeds the threshold of 20). Indeed, the simple estimator basically states that no reaction occurs between $t$ and $t+\mathrm{d} t$, the reactions being corrected over the coupling iterations. Consequently, the higher the amount of reacting material, the higher the number of iterations. When the reacting front changes cells, the amount of reaction is shared between the two cells, and since the system is solved globally, the coupling sees lower local 

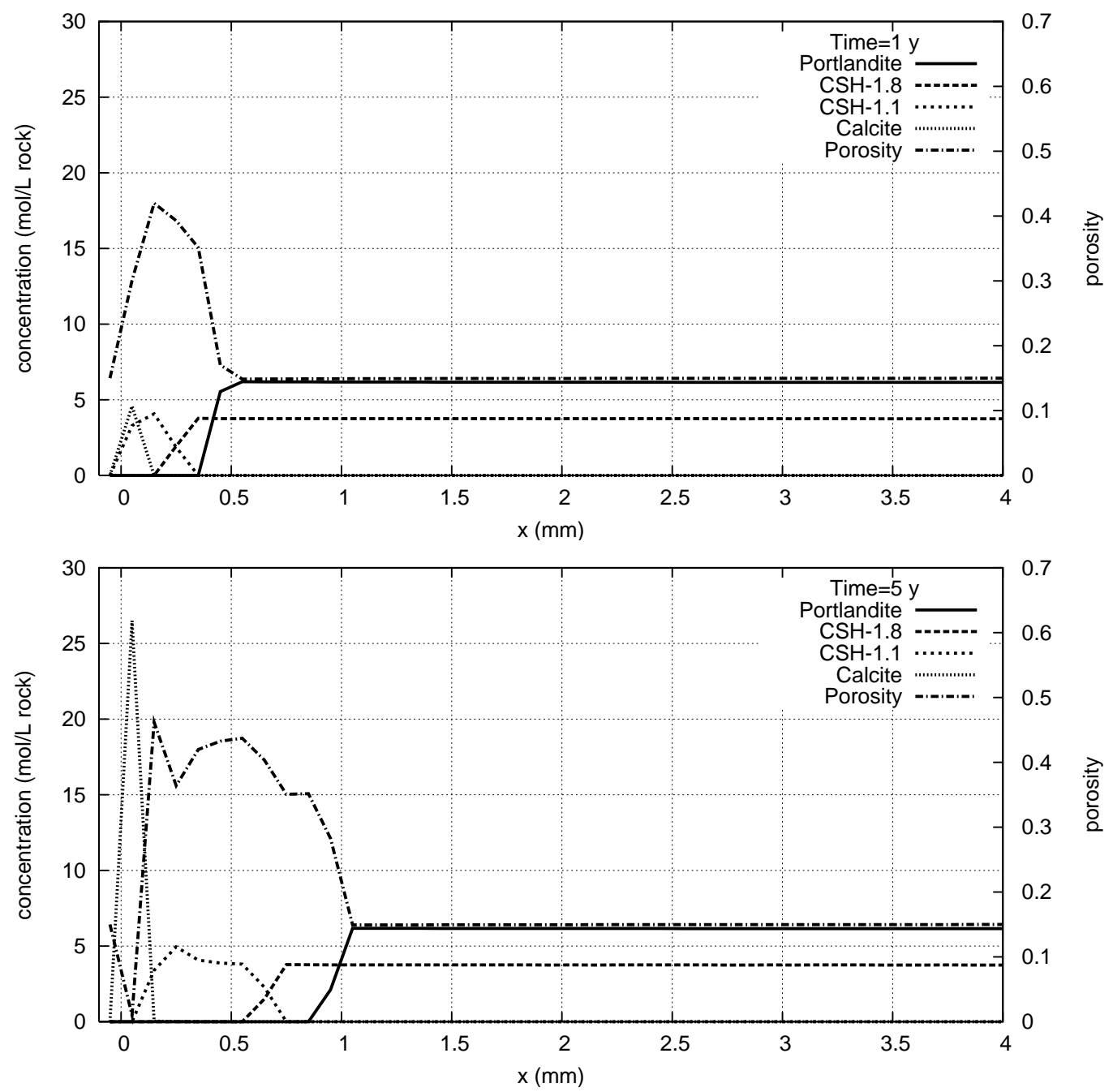

Fig. 7. Profiles of main mineral phases and porosity during degradation and carbonation of a cement. A sharp drop in the porosity is observed at the cement-fluid interface.

reacting quantities, so that the number of iterations is lower.

The observations are illustrated by Figure 8, i.e. the cumulative number of iterations required to complete the simulation. Note that the cumulative number of iterations for the simple estimator soars when sealing becomes effective at the interface. Not only does it require a large number of iterations per timestep, but the time-step size is also dramatically reduced due to the automatic time-step adjustment to keep the number of iterations at a reasonable level. With the higher performance of the improved estimator, no noticeable numerical difficulties emerge, even after nearly complete clogging. Moreover, it is interesting to note that, as diffusivity drops by several orders of magnitude at the interface, the cement becomes protected from further degradation, leading to a strong decrease in hydroxide release. The system becomes almost completely sealed. The remaining degradation of the portlandite creates a rise 


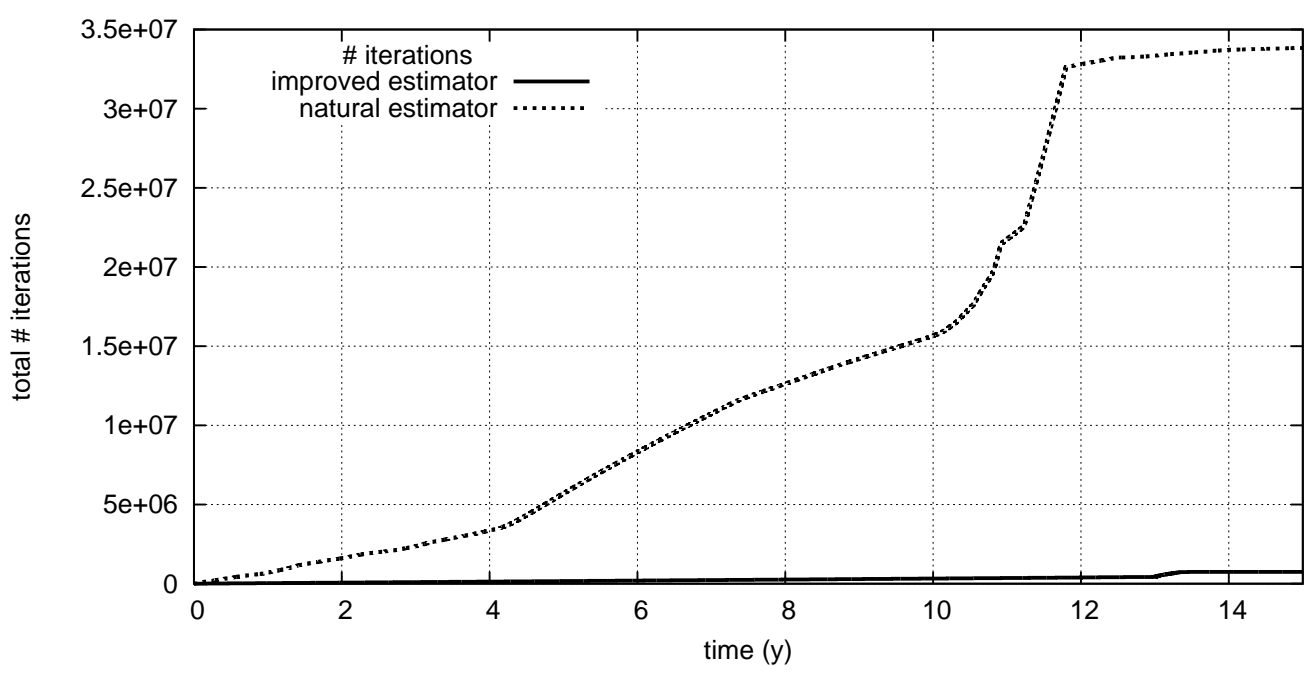

Fig. 8. Number of iterations required to solve the cement carbonation problem for two types of estimators: cumulative iterations during the simulation for the natural and the improved estimators.

in the $\mathrm{Ca} / \mathrm{Si}$ ratio of the previously hydrolyzed $\mathrm{CSH}$ until full equilibrium is reached. Subsequently, a limited form of self-healing is predicted by the modeling (Fig. 9).

\section{Conclusions}

Scientists involved in reactive transport modeling are always confronted by a trade-off between accuracy and efficiency. This paper deals with both aspects, proposing novel analytical solutions for strongly reactive test-cases with variations of porosity due to mineral dissolution and/or precipitation. The testcases can be simulated with any reactive transport model. The application of HYTEC, which uses implicit and explicit methods to deal with porosity variations, leads to the unambiguous conclusions that only the implicit implementation produces accurate solutions for any time-step size. Implicit methods, however, are CPU consuming, hence optimization remains a critical issue.

The operator-splitting method has several interesting advantages provided iterations are used to achieve an accurate solution. The better the estimate of the unknowns (e.g. immobile fractions) at the beginning of each time-step, the less iterations are required. Instead of estimating the unknowns based on the results of the previous time-step, we propose to estimate the unknowns by the values which they are predicted to reach at the end of the next time-step. The method, which is simple and based on the simulation history only, leads to a dramatic decrease in the number of iterations required for each time-step: a single iteration is often enough. It is worth noting that although developed 

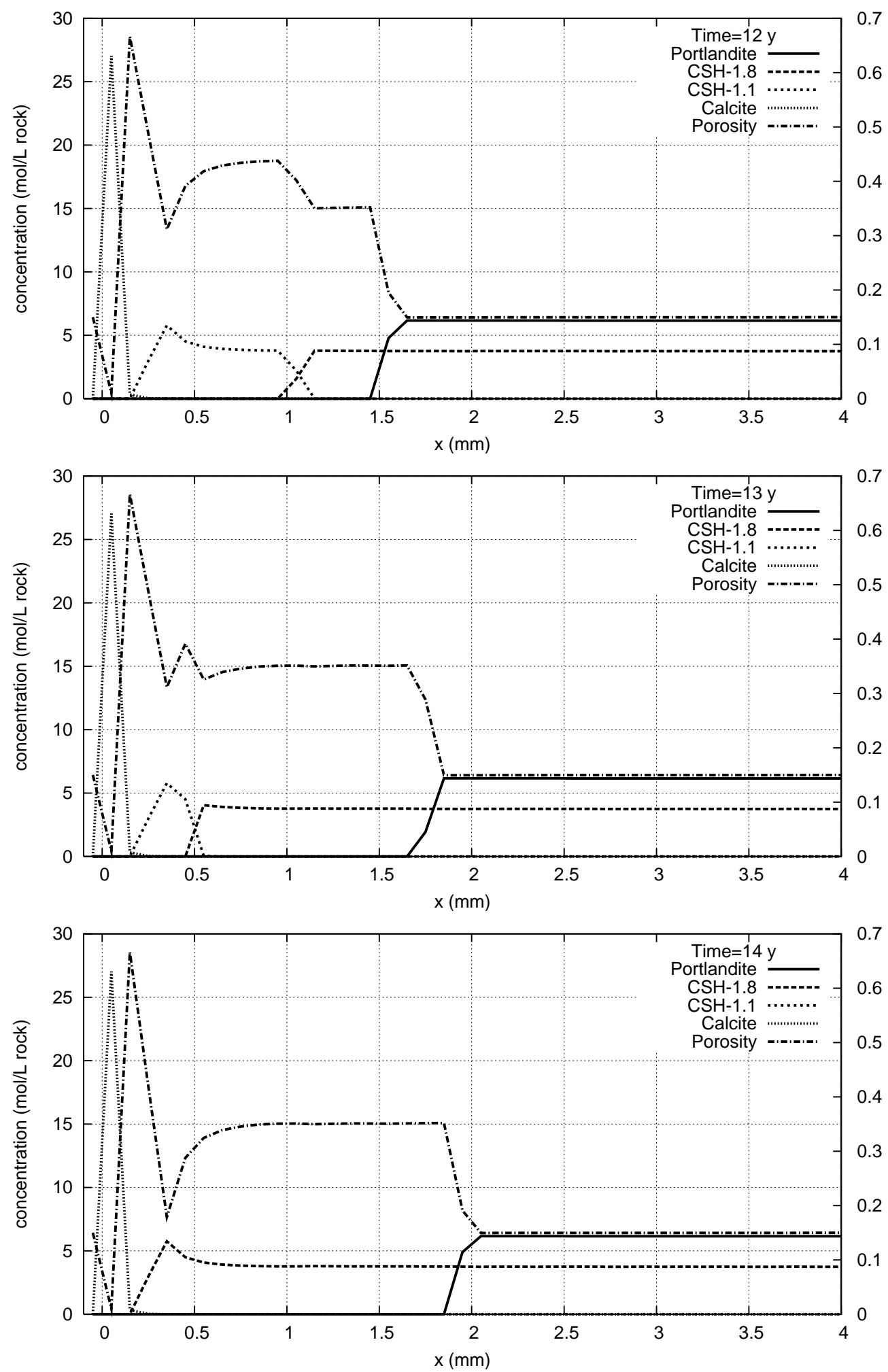

Fig. 9. Simulation of CEM I cement carbonation, illustrating profiles of minerals and porosity and a partial self-healing of the cement due to the formation of a calcite crust. 
along a specific simple test-case, the efficiency is still improved for applications on more complex systems, including several sharp minerals fronts, or smoother fronts due to sorption or kinetics.

The new estimation method has been implemented in HYTEC and applied to many different test cases, among which a few are outlined in this paper. It is observed that the convergence rate is improved for nearly all cases, whatever reaction processes are involved. HYTEC sometimes reduces the timestep when it encounters stiff or numerically difficult points in the simulation. At these specific moments, the conservative estimator is often more efficient. Hence, the model should be able to detect these moments and adapt the estimation method as a function of these events.

\section{Acknowledgment}

The authors acknowledge the Ple Gochimie Transport for its financial support to the development of the HYTEC code. The authors are also indebted to two anonymous reviewers for their constructive and detailed comments which greatly helped improve the quality of the paper.

\section{A Analytical resolution of the reaction only test-case}

The reaction only test-case is described by the following equations:

$$
\left\{\begin{aligned}
\frac{\mathrm{d} \omega c}{\mathrm{~d} t} & =-\frac{\mathrm{d} \omega \bar{c}}{\mathrm{~d} t} & & \text { (conservation of mass) } \\
& =\frac{1}{V_{m}} \frac{\mathrm{d} \omega}{\mathrm{d} t} & & \text { (conservation of volume) }
\end{aligned}\right.
$$

The system of equations, and its subsequent resolution, would still hold in a stationary unsaturated case: porosity should then be multiplied by the reduced water saturation in all terms of the equation. The resolution of this case is obtained in several steps. First, equation A.1 shows that $\omega \bar{c}$ is a function of $\omega$ only, given by an ordinary differential equation (ODE). Second, by developing the $\omega c$ time derivative, it is possible to separate $c$ and $\omega$ in a second ODE. The two equations are readily solved:

$$
\begin{aligned}
\omega(t) & =\omega_{0} \frac{1-c_{0} V_{m}}{1-c V_{m}} \\
\omega(t) \bar{c}(t) & =\omega_{0} \bar{c}_{0}-\frac{\omega-\omega_{0}}{V_{m}} \quad\left(\mathrm{~mol} \cdot \mathrm{L}^{-1} \text { medium }\right)
\end{aligned}
$$


Finally, the aqueous concentration $c$ can be expressed in an ODE, by substituting the kinetic law in the system, and eliminating the porosity $\omega$. We then obtain the following reduced equation:

$$
\frac{\mathrm{d} c}{\mathrm{~d} t}=\frac{k S_{s}(\omega)}{\omega}\left(1-V_{m} c\right)(1-c / K)
$$

By using a very simple law to describe the variation of the specific surface area, i.e. $S_{s}(\omega)=\omega S_{0}$, we can obtain the following solution:

$$
\begin{aligned}
c(t) & =\frac{1 / V_{m}-K \zeta(t)}{1-\zeta(t)} \\
\text { where } \zeta(t) & =\frac{c_{0}-1 / V_{m}}{c_{0}-K} \exp \left[-\frac{k}{K}\left(K V_{m}-1\right) S_{0} t\right] \\
S_{s}(\omega) & =\omega S_{0}
\end{aligned}
$$

\section{B Analytical resolution of the reactive transport test-case}

The system follows the system of equations:

$$
\left\{\begin{array}{l}
\frac{\partial \omega c}{\partial t}=\frac{\partial}{\partial x}\left(D_{e}(\omega) \frac{\partial c}{\partial x}\right)-\frac{\partial \omega \bar{c}}{\partial t} \\
\frac{\partial \omega \bar{c}}{\partial t}=-k S(\omega)\left(1-\frac{c}{K}\right)=-\frac{1}{V_{m}} \frac{\partial \omega}{\partial t}
\end{array}\right.
$$

As in the previous problem, we need to inject information relative to the evolution of the pore structure. To keep the system simple, we used $S(\omega)=$ $\omega S_{0}$ and $D_{e}(\omega)=\omega D_{0}$. Accordingly, the system can be rewritten as follows:

$$
\left\{\begin{aligned}
\omega \frac{\partial c}{\partial t}+c \frac{\partial \omega}{\partial t} & =\omega D_{0} \frac{\partial^{2} c}{\partial x^{2}}+D_{0}\left(\frac{\partial \omega}{\partial x}\right)\left(\frac{\partial c}{\partial x}\right)+\frac{1}{V_{m}} \frac{\partial \omega}{\partial t} \\
\frac{\partial \omega}{\partial t} & =k V_{m} S_{0} \omega\left(1-\frac{c}{K}\right)
\end{aligned}\right.
$$

The first equation is transformed by gathering the differential terms for concentration on the one hand and porosity on the other hand, then by substituting the second equation in the final term for porosity. This leads to:

$$
\omega \frac{\partial c}{\partial t}=\omega D_{0} \frac{\partial^{2} c}{\partial x^{2}}+D_{0}\left(\frac{\partial \omega}{\partial x}\right)\left(\frac{\partial c}{\partial x}\right)+k V_{m} S_{0} \omega\left(\frac{1}{V_{m}}-c\right)\left(1-\frac{c}{K}\right)
$$

Let us assume that the cross-term of the derivative is negligible:

$$
D_{0}\left(\frac{\partial \omega}{\partial x}\right)\left(\frac{\partial c}{\partial x}\right) \approx 0
$$


This is certainly true at the very beginning of the porosity evolution, when its spatial gradient is still small - an assumption which has to be verified $a$ posteriori. Accordingly, the system can be simplified as follows:

$$
\left\{\begin{array}{l}
\frac{\partial c}{\partial t}=D_{0} \frac{\partial^{2} c}{\partial x^{2}}+k V_{m} S_{0}\left(\frac{1}{V_{m}}-c\right)\left(1-\frac{c}{K}\right) \\
\frac{\partial \omega}{\partial t}=k V_{m} S_{0} \omega\left(1-\frac{c}{K}\right)
\end{array}\right.
$$

The second equation is a straightforward ODE of $c$, which is solved straightforwardly. In the first equation, the porosity has completely disappeared. Note that the obtained partial derivative equation is close to the heat equation, with a particular solution $c_{\infty}(x, t)$, i.e. an associated homogeneous equation. The reduced equation is solved by using its eigenvalues composed in a Fourier series (7). Finally, initial and boundary conditions constrain the parameters of the Fourier series. The solution for the system becomes:

$$
\left\{\begin{aligned}
c(x, t)= & c_{\infty}(x)-\sum_{n=1}^{\infty} b_{n} \sin \left(\frac{n \pi}{L} x\right) e^{-\left(D_{0} \frac{n^{2} \pi^{2}}{L^{2}}+k S_{0} / K\right) t} \\
\omega(x, t)= & \omega_{0} \exp \left[k S_{0} V_{m} / K\left(\left(K-c_{\infty}(x)\right) t \ldots\right.\right. \\
& \left.\left.-\sum_{n=1}^{\infty} \frac{b_{n}}{D_{0} \frac{n^{2} \pi^{2}}{L^{2}}+k S_{0} / K} \sin \left(\frac{n \pi}{L} x\right) e^{-\left(D_{0} \frac{n^{2} \pi^{2}}{L^{2}}+k S_{0} / K\right) t}\right)\right]
\end{aligned}\right.
$$

with

$$
\left\{\begin{aligned}
c_{\infty}(x)= & A e^{\Omega x}+B e^{-\Omega x}+K \\
A= & \frac{c_{0}-K}{1-e^{2 \Omega L}} \\
B= & \frac{c_{0}-K}{1-e^{-2 \Omega L}} \\
\Omega= & \sqrt{k S_{s_{0}} /\left(K D_{0}\right)} \\
\forall n \in \mathcal{N}^{*}, b_{n}= & -2 \frac{K-c_{0}}{n \pi}\left[(-1)^{n}-1\right] \ldots \\
& -\frac{2 n \pi}{\Omega^{2} L^{2}+n^{2} \pi^{2}}\left[A\left(e^{\Omega L}(-1)^{n}-1\right)+B\left(e^{-\Omega L}(-1)^{n}-1\right)\right]
\end{aligned}\right.
$$

The series are absolutely convergent since the coefficients $b_{n}$ accept an equivalent in $\zeta / n$. The terms of the series therefore have an equivalent in $\frac{\xi}{n^{3}} e^{-\sigma n^{2} t}$; a very fast convergence is thus guaranteed, especially for large values of time $t$.

For cases where a stationary state of the aqueous species is rapidly reached (compared to the characteristic time of mineral and porosity variations), the 
solution can be asymptotically simplified:

$$
\left\{\begin{array}{l}
c(x, t)=c_{\infty}(x) \\
\omega(x, t)=\omega_{0} \exp \left[-V_{m} k S_{0} / K\left(A e^{\Omega x}+B e^{-\Omega x}\right) t\right]
\end{array}\right.
$$

\section{References}

[1] Adenot F., M. Buil (1992) Modelling of the corrosion of the cement paste by deionized water, cement and concrere research, 22, 489-496.

[2] Archie G.E. (1942) The electrical resistivity log as an aid to determining some reservoir characteristics, Transactions of the American Institute of Mining Engineers, 146, 54-61.

[3] Badouix F., P. Le Bescop, P. Lovera, F. Adenot, J.P. Bournazel (1998) Carbonatation dune pâte de CPA-CEM I au cours dun essai de lixiviation à $\mathrm{pH}$ de 8.5 et modélisation dun cas simplifié de carbonatation, XVIèmes Rencontres Univ. Génie Civil OE98, Reims, France vol I.

[4] Bear J. (1972) Dynamics of fluids in porous media, Dover Publications Inc., New York.

[5] Carman P.C. (1937) Fluid through granular beds, transactions Institution of Chemical Engineers, 15, 150-166.

[6] Carrayrou J., R. Mosé, P. Behra (2004) Operator-splitting procedures for reactive transport and comparison of mass balance errors, Journal of Contaminant Hydrology, 68, 239-268.

[7] Carslaw H.S., J.C. Jaeger (1959) Conduction of heat in solids, Oxford Science Publications, New York, second edition.

[8] Charlet L., S. Chakraborty, C.A.J. Appelo, G. Roman-Ross, B. Nath, A.A. Ansari, M. Lanson, D. Chatterjee, S. Basu Mallik (2007) Chemodynamics of an arsenic hotspot' in a West Bengal aquifer: A field and reactive transport modeling study, Applied Geochemistry, 22, 1273-1292.

[9] Chilingarian G.V., J. Torabzadeh, H.H. Rieke, M. Metghalchi, S.J. Mazzullo (1992) Interrelationships among surface area, permeability, porosity, pore size and residual water saturation, Developments in Petroleum Science, 30, 379-397.

[10] de Marsily G. (1986) Quantitative Hydrogeology: groundwater hydrology for engineers, Academic Press, Inc.

[11] De Windt L., F. Marsal, E. Tinseau, D. Pellegrini (2008) Reactive transport modeling of geochemical interactions at a concrete/argillite interface, Tournemire site (France), Physics and Chemistry of the Earth, 33, 295-305.

[12] De Windt L., R. Badreddine, V. Lagneau (2007) Long-term reactive transport modelling of stabilised/solidified waste: from dynamic leaching tests to disposal scenarios, Journal of Hazardous Materials, 139, 529-536.

[13] De Windt L., H. Schneider, C. Ferry, H. Catalette, V. Lagneau, C. 
Poinssot, A. Poulesquen, C. Jegou (2006) Modeling radionuclide sourceterms and release in a spent nuclear fuel disposal, Radiochimica Acta, 94, 787-794.

[14] Gherardi F., T. Xu, K. Pruess (2007) Numerical modeling of self-limiting and self-enhancing caprock alteration induced by CO2 storage in a depleted gas reservoir, Chemical Geology, 244, 103-129.

[15] Kohfahl C., P.L. Brown, C.M. Linklater, K. Mazur, P. Irannejad, A. Pekdeger (2008) The impact of pyrite variability, dispersive transport and precipitation of secondary phases on the sulphate release due to pyrite weathering, Applied Geochemistry, 23, 3783-3798.

[16] Kieffer B., C.F. Jové, E.H. Oelkers, J. Schott (1999) An experimental study of the reactive surface area of the Fontainebleau sandstone as a function of porosity, permeability, and fluid flow rate, Radiochimica et cosmochimica acta, 63, 3225-3534.

[17] Lagneau V. (2000) Influence des processus géochimiques sur le transport en milieu poreux; application au colmatage de barrières de confinement potentielles dans un stockage en formation géologique, Ph.D. thesis École des Mines de Paris, $181 \mathrm{p}$.

[18] Lichtner P.C., C. Steefel, E. Oelkers editors (1996) Reactive transport in porous media, chapter 1 Continuum formulation of multicomponentmiltiphase reactive transport, Reviews in mineralogy, 34, 1-81.

[19] Lichtner P.C. (1992) Time-space continuum description of the fluid/rock interaction in permeable media, Water resources research, 28, 3135-3155.

[20] MacQuarrie K.T.B., K.U. Mayer (2005) Reactive transport modeling in fractured rock: A state-of-the-science review, Earth-Science Reviews, 72, 189-227.

[21] Saaltink M.W., J. Carrera, C. Ayora (2001) On the behavior of approaches to simulate reactive transport, Journal of Contaminant Hydrology, 48, 213-235.

[22] Samper J., C. Lu, L. Montenegro (2008) Reactive transport model of interactions of corrosion products and bentonite, Physics and Chemistry of the Earth, Parts A/B/C, 33, Supplement 1, Clays in Natural \& Engineered Barriers for Radioactive Waste Confinement, S306-S316.

[23] Spiteri C., C.P. Slomp, P. Regnier, C. Meile, P. Van Cappellen (2007) Modelling the geochemical fate and transport of wastewater-derived phosphorus in contrasting groundwater systems, Journal of Contaminant Hydrology, 92, 87-108.

[24] Steefel C.I., A.C. Lasaga (1994) A coupled model for transport of multiple chemical species and kinetic precipitation/dissolution reactions with applications to reactive flow in single phase hydrothermal system, American Journal of Science, 294, 529-592.

[25] Trotignon L., V. Devallois, H. Peycelon, C. Tiffreau, X. Bourbon (2007) Predicting the long term durability of concrete engineered barriers in a geological repository for radioactive waste, Physics and Chemistry of the Earth, 32, 259-274. 
[26] van der Lee J., L. De Windt, V. Lagneau (2008) Application of reactive transport models in cement-based porous media", Concrete Modelling CONMOD '08, May 26-28, Delft, The Netherlands. Proceedings of the International RILEM Symposium, Eds. E. Schlangen and G. De Schutter, 463-470.

[27] van der Lee J., L. De Windt, V. Lagneau, P. Goblet (2003) Moduleoriented modeling of reactive transport with HYTEC, Computers $\&$ Geosciences, 29, 265-275.

[28] Wagner R., M. Kuhn, V. Meyn, H. Pape, U. Vath, C. Clauser (2005) Numerical simulation of pore space clogging in geothermal reservoirs by precipitation of anhydrite, International Journal of Rock Mechanics and Mining Sciences, 42, Rock Physics and Geomechanics, 1070-1081.

[29] Yeh G., V. Tripathi (1991) A model for simulating transport of reactive multi-species components: model development and demonstration, Water Resources Research, 27, 3075-3094. 